\title{
Impact of a national audit project on gynaecologists in Scotland
}

\author{
Gillian C Penney, Allan Templeton
}

\begin{abstract}
The objectives of the study were $(a)$ to determine consultant gynaecologists' awareness of and views on a national audit project (the gynaecology audit project in Scotland) and (b) to measure changes in their reported practice in relation to 12 specific elements of care related to three audit topics (induced abortion, endometriosis, and vulvar carcinoma) for which recommendations for change had been made within the project. The study comprised a postal questionnaire survey of all 128 consultant gynaecologists in NHS practice in Scotland. The response rate was $90 \%$. Of the respondents, $96 \%$ (109/113) recalled receiving feedback material from the audit project team and around $75 \%$ (range $66 / 89$ to $84 / 105$ ) had retained feedback reports for future reference. For the two more common clinical topics (induced abortion and endometriosis), over two thirds of the respondents indicated that they had been prompted to reconsider or change aspects of practice. Significant changes in reported practice, in line with project recommendations, were found for seven of the 12 specific elements of care examined. Thus, gynaecologists in Scotland showed a high level of awareness of and positive views towards a national audit project. Significant changes in reported practice, in accordance with circulated recommendations, were measurable in relation to several elements of clinical care.
\end{abstract}

(Quality in Health Care 1995;4:37-39)

Keywords: evaluation of audit, gynaecologists, change in clinical practice.

\section{Introduction}

The government's white paper Working for Patients of 1989 required that all doctors participate in medical audit. ${ }^{1}$ In the three years since the white paper, an estimated $£ 140 \mathrm{~m}$ has been invested in medical audit programmes in England alone. ${ }^{2}$ Widespread scepticism exists about whether this massive investment has resulted in tangible benefits to patients and in measurable changes in clinicians' practice. There is growing awareness of the need for evaluation of audit activities, or "audit of audit."3
The gynaecology audit project in Scotland was initiated by the Scottish executive committee of the Royal College of Obstetricians and Gynaecologists, and over the past two years it has conducted national audits of three gynaecological topics (induced abortion, endometriosis, and vulvar carcinoma). We assessed the impact of this audit project on gynaecologists' views and practice by means of a follow up survey.

\section{Methods}

The audit of each of the three topics had incorporated four stages.

Firstly, standards were set by agreement of a list of criteria for good quality care based on literature review, panel discussions, and consensus surveys, as previously described. ${ }^{4}$ Twenty criteria were agreed for induced abortion, 17 for endometriosis, and 15 for vulvar carcinoma.

Secondly, gynaecologists' reported practice was audited by means of questionnaire surveys on each of the three topics. These surveys were undertaken between September 1992 and March 1993 and covered all consultant gynaecologists in post in Scotland at the relevant times. For induced abortion and endometriosis 132 gynaecologists were surveyed and 121 responded $(92 \%$ response rate) and for vulvar carcinoma 126 consultants were surveyed and 120 responded (95\% response rate). The results of these exercises have been published elsewhere..$^{5-7}$

Thirdly, current practice was audited by means of a review of case notes undertaken in 10 representative hospitals throughout Scotland. ${ }^{8}$

Finally, the results of the audits and the recommendations for change were disseminated by circulating detailed reports to all consultant gynaecologists and by means of presentations at hospital based and national meetings.

In January 1994 a follow up questionnaire was sent to all 128 consultant gynaecologists then in post in Scotland. The mailing list was based on names and addresses obtained from the royal college and was updated by telephoning all the gynaecology units in Scotland. The aims of the questionnaire were to assess gynaecologists' awareness of the gynaecology audit project in Scotland, their views on the feedback reports circulated by the project team, and their perceptions as to 


\section{Criteria for good quality care addressed in follow up questionnnaire ${ }^{\star}$}

Induced abortion

- Doctors managing induced abortion should have a policy for identifying and treating women at risk of genital tract infection

- In selected women undergoing surgical abortion a technique for cervical predilatation should be used

- For second trimester medical abortion, the addition of mifepristone to prostaglandin regimens reduces the time between induction and abortion

- Before she is discharged each patient should have agreed on a contraceptive plan and should have been offered contraceptive supplies

- A follow up appointment, either at the hospital or with the referring doctor, should be offered to every patient

Endometriosis

- At diagnostic laparoscopy a double puncture technique should be used to permit the entry of instruments for manipulation of the pelvic organs

- At diagnostic laparoscopy the description of pelvic findings recorded should permit staging of the disease into minimal, mild, moderate, and severe categories

- The description should be supplemented by a diagram or photograph

- For infertile patients with mild disease, the first line treatment is "expectant management"

- When medical treatment is chosen for endometriosis the preferred first line treatment is continuous progestogen

Vulvar carcinoma

- Vulvar carcinoma should not be managed by the "occasional oncologist" alone but by a recognised individual in each hospital or district who has a subspecialty interest

- Regular, long term follow up should take place at a special multidisciplinary gynaecological oncology clinic

* These criteria were based on literature review and were validated by a consensus of agreement among Scottish gynaecologists ${ }^{4-7}$

whether the project had influenced their practice.

In addition, 12 criteria for good quality care were selected from among the $\mathbf{5 2}$ agreed for the three audit topics as being of particular importance or as representing aspects of practice where changes were required. These 12 criteria are listed in the box. Inquiry was made into practice relating to these criteria for comparison with responses made to similar inquiries in the original questionnaire surveys. ${ }^{5-7}$

A second copy of the questionnaire with a letter of reminder was sent to gynaecologists who did not respond within two weeks.

Data from the follow up questionnaires were entered into a purpose designed database using Paradox (Borland) software on an IBM compatible personal computer. Significance testing using the McNemar test was performed using the Instat 2 (Graphpad Software) package.

\section{Results}

Of the 128 questionnaires sent out, 115 were eventually returned, completed, to the project office - a response rate of $90 \%$. Of these, 113 were returned in time for analysis. Of these 113 responders, 109(96\%) recalled receiving one or more of the feedback reports circulated by the project team. Of those who recalled receiving them, $80 \%$ ( 84 out of 105 ), $78 \%$ (80

Responding gynaecologists who were prompted to change or reconsider their practice after receiving audit feedback reports

\begin{tabular}{lcccc} 
& & \multicolumn{3}{c}{ No (\%) prompted to: } \\
\cline { 3 - 5 } Topic & $\begin{array}{l}\text { No who recalled } \\
\text { receiving reports }\end{array}$ & Change practice & Reconsider practice & $\begin{array}{c}\text { Change or } \\
\text { reconsider practice }\end{array}$ \\
\hline Induced abortion & 105 & $29(28)$ & $41(39)$ & $70(67)$ \\
Endometriosis & 102 & $14(14)$ & $56(55)$ & $70(69)$ \\
Vulvar cancer & 89 & $4(4)$ & $22(25)$ & $26(29)$ \\
\hline
\end{tabular}

out of 102), and $74 \%$ (66 out of 89 ) indicated that they had retained for future reference the reports on abortion, endometriosis, and vulvar cancer respectively.

Consultants were asked how interesting and useful they had found the feedback reports. Responses are summarised in the table. For abortion and endometriosis, over two thirds of responders were prompted to change or, at least reconsider, aspects of their practice. Only three gynaecologists indicated that they thought that the reports were "a waste of paper."

Ninety four consultants $(88 \%$ of those answering) thought that it would be worth while for the project team to address further topics in a similar manner, and 59 of them suggested 96 suitable topics. The suggestions encompassed almost the whole of gynaecological (and indeed, obstetric) practice. The most popular suggestions were menorrhagia and dysfunctional uterine bleeding (22 consultants), incontinence (14 consultants), and infertility (12 consultants).

For each of the 12 specific items of care chosen to be addressed in the follow up survey, the responses of those consultants who answered the relevant questions in both the initial and follow up questionnaires were compared using the McNemar test.

\section{INDUCED ABORTION}

Three significant changes, in line with recommendations, occurred. Firstly, more consultants indicated that they would predilate the cervix at relatively advanced gestations (75 $v 60$ out of 89 ); 19 did so who had not done so before and four had changed their practice in the opposite direction $\left(\chi^{2}=8 \cdot 5, p=0.003\right)$. Secondly, more consultants would use mifepristone before a second trimester medical 
abortion (59 $v 18$ out of 89 ); 41 did so who had not done so before and none had changed their practice in the opposite direction $\left(\chi^{2}=36\right.$, $\mathrm{p}<0.0001)$. Finally, more consultants adopted a policy of universal bacteriological screening or antibiotic prophylaxis for managing potential genital tract infection (33 $v 23$ out of $90) ; 12$ did so who had not done so before and two had changed their practice in the opposite direction $\left(x^{2}=5.78, p=0.016\right)$. The difference in the numbers who would offer contraceptive supplies as well as advice (51 $v 43$ out of 88) and who would offer some form of follow up (65 $v 64$ out of 87 ) failed to reach significance.

\section{ENDOMETRIOSIS}

Significantly more gynaecologists indicated that they now usually used a diagram to help record the findings at diagnostic laparoscopy (59 $v 44$ out of 100); 22 now did so who had not done so before and seven had changed their practice in the opposite direction $\left(\chi^{2}=6.76\right.$, $\mathrm{p}=0.009)$. Similarly, more would choose a progestogen as first line medical treatment (37 $v 26$ out of 101); 13 would choose progestogen who had not done so before and two had changed their practice in the opposite direction $\left(\chi^{2}=6 \cdot 67, p=0 \cdot 01\right)$.

Fewer gynaecologists would now use inappropriate drug treatments for infertile women with mild endometriosis (28 $v 50$ out of 95$) ; 25$ who previously would have prescribed drug treatments would no longer do so and three had changed practice in the opposite direction $\left(\chi^{2}=15 \cdot 7, \mathrm{p}<0.0001\right)$. The differences in numbers who indicated that they always use a double puncture technique at diagnostic laparoscopy (62 $v 59$ out of 102) and who used the revised American Fertility Society scoring system for staging (15 $v 15$ of 102) failed to reach significance.

\section{VULVAR CARCINOMA}

Significantly more gynaecologists would now offer follow up at a special, multidisciplinary clinic (32 $v 23$ out of 58); 11 would do so who did not before and two had changed their practice in the opposite direction $\left(\chi^{2}=4.92\right.$, $p=0.026)$. There was no difference in the number of gynaecologists who were not specialists in oncology who would manage cases of vulvar cancer themselves ( $3 v 2$ out of 40).

\section{Discussion}

The follow up survey showed a high level of awareness among gynaecologists of a national audit project initiated by the Scottish branch of their royal college. Most valued the feedback reports circulated by the project team sufficiently to retain them for future reference and, for the two more common clinical topics, over two thirds indicated that they were prompted to reconsider or change their practice. The theoretical advantages of multidisciplinary, clinical (rather than medical) audit are acknowledged, but this confidential, single discipline exercise seemed to engender a strong sense of ownership and cooperation. In future, however, this approach to audit might be expanded to include junior medical staff and referring general practitioners.

The finding of a significant increase over the timescale of the project in the number of consultants whose practice accords with the audit recommendations for seven of the 12 specific elements of care studied confirms respondents' expressed views that they were prompted to change their practice. Reported practice may not necessarily reflect actual practice, but in this project the impressions of current care gained from the questionnaire audit and the review of case notes were similar. $^{58}$

The gynaecology audit project in Scotland has shown that Scottish gynaecologists are interested and willing to participate in medical audit. Refinements in audit methods must be sought to translate this evident good will into more substantial improvements in patient care.

We thank Drs A Glasier, M Hall, S Prigg, J Grant, and C West for advice on the content of the questionnaire. Mrs Elaine Stirton typed the manuscript and also computerised the data. The gynaecology audit project in Scotland is funded by a gran from the Clinical Resource and Audit Group of the Scottish Office Home and Health Department (but the views expressed are those of the authors and not necessarily those of the group or the department). We particularly thank the gynaecologists in Scotland who responded to our follow up questionnaire.

1 Department of Health Working for patients. Medical Audit. London: HMSO, 1989.

2 Department of Health. Medical audit in the hospital and community health services. London: $\mathrm{DoH}, 1991$.

3 Walshe K, Coles J. Medical audit: in need of evaluation Quality in Health Care 1993;2:189-90.

4 Penney GC, Glasier A, Templeton A. Agreeing criteria for audit of the management of induced abortion: an approach by national consensus survey. Quality in Health Care 1993;2:167-9.

5 Penney GC, Templeton A. Induced abortion: an audit of reported current practice among consultant gynaecologists in Scotland. $\mathrm{Br} 7 \mathrm{Obstet}$ Gynaecol 1994 101:523-8.

6 Penney GC, Templeton A. The management of endometriosis: an audit of reported current practice among criosis: an audit of reported current practice among 1994;52:165-74.

7 Penney GC, Kitchener HC, Templeton A. The management of carcinoma of the vulva: current opinion and ment of carcinoma of the vulva: current opinion and current practice among consultant gy
Scotland. Health Bull (Edinb) (in press).

8 Penney GC, Glasier A, Templeton A. A multicentre, criterion based audit of the management of induced abortion in Scotland. BMF 1994;309:15-9. 